\title{
Identification and profiling of Manduca sexta microRNAs and their possible roles in regulating specific transcripts in fat body, hemocytes, and midgut $\hbar$
}

\author{
Xiufeng Zhang ${ }^{1}$, Yun Zheng ${ }^{2}$, Xiaolong $\mathrm{Cao}^{1,3}$, Ren Ren ${ }^{4}$, Xiao-Qiang $\mathrm{Yu}^{5}$, Haobo Jiang ${ }^{1 *}$ \\ ${ }^{1}$ Department of Entomology and Plant Pathology, Oklahoma State University, \\ Stillwater, OK 74078, USA \\ ${ }^{2}$ Faculty of Life Science and Technology, Kunming University of Science and Technology, \\ Kunming, Yunnan 650500, P.R. China \\ ${ }^{3}$ Department of Biochemistry and Molecular Biology, Oklahoma State University, \\ Stillwater, OK 74078, USA \\ ${ }^{4}$ School of Life Sciences, Fudan University, Shanghai 200433, P.R. China \\ ${ }^{5}$ Division of Molecular Biology and Biochemistry, University of Missouri-Kansas City, \\ Kansas City, MO 64110, USA
}

Running title: Manduca sexta microRNA identification and tissue-specific regulation

Key words: innate immunity; insecticide toxicity; metabolism; mitochondrial miRNA regulation; target site prediction; Illumina sequencing.

Abbreviations: ABC transporter, ATP-binding cassette transporter; ACBP, acyl-CoA-binding protein; ADCY, adenylate cyclase; ADGF, adenosine deaminase-related growth factor; $\mathrm{ADK}$, adenylate kinase; AKH, adipokinetic hormone; AMP, antimicrobial peptide; ARF, ADP-ribosylation factor; $\mathrm{CCDC}$, coiled-coil domain-containing protein; $\mathrm{CF}$, control fat body; $\mathrm{CH}$, control hemocytes; CoA, coenzyme A; DsCAM, Down syndrome cell adhesion molecule; eIF, eukaryotic translation initiation factor; FAMeT, farnesoic acid $O$ methyltransferase; Fkbp, FK509 binding protein; FMO, flavin-dependent monooxygenase; FPKM, fragments per kilobase of transcript per million mapped reads; GP, gut protease; HP, hemolymph protease; HSP, heat shock protein; IF, induced fat body; IH, induced hemocytes; IML, immulectin; JH, juvenile hormone; LAP, leucyl aminopeptidase; LPP, lipid phosphate phosphohydrolase; LRR, leucine-rich repeat protein; MCT, monocarboxylate transporter; MFE, minimal free energy; MSBP, membrane steroid 
binding protein; nt, nucleotide; PABP, polyadenylate binding protein; PAP, prophenoloxidase activating proteinase; proPO, prophenoloxidase; RISC, RNA induced silencing complex; SCOT, succinyl-CoA: 3-ketoacid CoA transferase; serpin, serine proteinase inhibitor; SSR, signal sequence receptor; TCA cycle, tricarboxylic acid cycle; $\mathrm{TF}$, transcription factor; UTR, untranslated region.

Send correspondence to:

Haobo Jiang

Department of Entomology and Plant Pathology

Oklahoma State University

Stillwater, OK 74078

Telephone: (405)-744-9400

Fax: (405)-744-6039

E-mail: haobo.jiang@okstate.edu

is While microRNA* naming is still used, their corresponding $-5 p$ or $-3 p$ names are posted on our website (http://entoplp.okstate.edu/profiles/profiles/jiang.htm).

E-mail addresses:

XZ: xiufeng.zhang@okstate.edu

YZ: zhengyun5488@,gmail.com

XC: xiaolong.cao@okstate.edu

RR: renren@,fudan.edu.cn

XY: yux@umkc.edu

HJ: haobo.jiang@okstate.edu 


\section{Abstract}

2 Significance of microRNA-mediated posttranscriptional regulation has been appreciated ever

3 since its discovery. In the tobacco hornworm Manduca sexta, 164 conserved and 16 novel

4 microRNAs have been identified experimentally (Zhang et al., 2012, 2014). To extend the list of

5 microRNAs in this lepidopteran model species and further explore their possible regulatory roles,

6 we constructed and sequenced small RNA libraries of $M$. sexta fat body, hemocytes and midgut,

7 since transcriptomes of these tissues from the $5^{\text {th }}$ instar larvae had been studied quite extensively.

8 Each library represented a mixture of the same tissues from larvae that were naïve or induced by

9 three different pathogens. From a total of 167 million reads obtained, we identified two new

10 variants of conserved miR-281 and miR-305 and six novel microRNAs. Abundances of all

11 microRNAs were normalized and compared to reveal their differential expression in these three

12 tissues. Star strands of ten microRNAs were present at higher levels than the corresponding

13 mature strands. From a list of tissue-specific transcripts, we predicted target sites in 3'-UTRs

14 using preferentially expressed microRNA groups in each tissue and suggested possible

15 regulatory roles of these microRNAs in energy metabolism, insecticide resistance, and some

16 mitochondrial and immune gene expression. Examining manifold targets, microRNA regulations

17 were suggested of multiple physiological processes. This study has enriched our knowledge of $M$.

18 sexta microRNAs and how microRNAs potentially coordinate different physiological processes.

\section{1. Introduction}

21 MicroRNAs (i.e. miRNAs), about $22 \mathrm{nt}$ long, post-transcriptionally regulate their target

22 mRNAs. Derived from direct transcripts or intron lariats, miRNA precursors exhibit canonical

23 stem-loop secondary structures (Asgari, 2011). Upon Dicer-1 cleavage, one strand of the miRNA

24 duplex (miRNA:miRNA*) is loaded into RISC to bind its target (typically in 3'-UTR of the 
1 mRNA) and executes translational repression or mRNA degradation. The passenger strand

2 miRNA*s usually exist at levels much lower than their corresponding mature strands, some

3 hardly detectable by deep sequencing. Under some circumstances, miRNA*s are maintained at

4 higher levels indicative of potential roles in regulating other genes (Etebari et al., 2013; Zhang et

5 al., 2014). The dominant usage of either 5p- or 3p-arms of miRNA precursors displays lineage

6 specificity implying a possible miRNA evolution mechanism (Marco et al., 2010) and is closely

7 associated with the physiological status of a specific tissue (Li et al., 2012b). In the past decades,

8 miRNAs are found to participate in the regulation of various physiological processes including

9 development, host defense, metabolism, and stress responses (Asgari, 2011; Baker and Thummel,

10 2007; Chawla and Sokol, 2011).

11 In insects, most miRNA studies are focused on development, tissue differentiation, and

12 miRNA biogenesis. Recent research uncovered miRNA regulation in defense against viruses,

13 bacteria, fungi, and apicomplexan parasites (Asgari, 2011; Hakimi and Cannella, 2011; Zhang et

14 al., 2014). Although metabolites were suggested to mediate insect immune reactions (Stanley et

15 al., 2009) and metabolism is essential to insect homeostasis, little is known on how miRNAs

16 may regulate insect metabolic processes. Hypometabolism is a common strategy of insects to

17 avoid environmental stresses like freezing and starvation. Possible regulatory pairs were

18 suggested of miRNAs and enzymes in the fatty acid biosynthesis and degradation pathways

19 based on evidence from natural models other than insects (Lyons et al., 2013a). Dysregulated

20 miRNAs were identified in the goldenrod gall fly Eurosta solidaginis during freezing and in the

21 red flour beetle Tribolium castaneum upon starvation, which were potentially linked to

22 hypometabolism (Courteau et al., 2012; Freitak et al., 2012; Lyons et al., 2013b). However, it is

23 unclear what targets those miRNAs might recognize. In Drosophila, several miRNAs (miR-8, -

$2414,-33$ and -278) modulate the balance of insulin signaling pathway and lipid storage (Baker and 
1 Thummel, 2007; Davalos et al., 2011; Hyun et al., 2009). Still, lipid metabolism is the only

2 process examined; more information is needed to fully appreciate miRNA regulation of

3 metabolism of carbohydrates, amino acids, and others. Intriguingly, miR-8 was also found to

4 regulate antimicrobial peptide production in D. melanogaster and Plutella xylostella (Choi and

5 Hyun, 2012; Etebari and Asgari, 2013). By examining manifold targets of one miRNA, it is

6 possible to discover more miRNA regulatory correlations between immunity and metabolism in

7 insects.

8 The tobacco hornworm, Manduca sexta, has been utilized as a biochemical model to study

9 insect physiological processes, like hormonal control, innate immunity, cuticle formation, and

10 neural development (Hiruma and Riddiford, 2010). Fat body and hemocytes are major sources of

11 plasma proteins while fat body and midgut are crucial for metabolism. M. sexta fat body and

12 hemocytes have been extensively investigated to understand their roles in immune responses

13 upon bacteria injection into the $5^{\text {th }}$ instar larvae. The quantitative transcriptome studies of fat

14 body and hemocytes have uncovered differentially expressed proteins likely involved in

15 immunity (Gunaratna and Jiang, 2013; Zhang et al., 2011; Zou et al., 2008). The analysis of $M$.

16 sexta midgut transcriptome revealed enzymes involved in digestion, detoxification and immunity

17 (Pauchet et al., 2010). With the genome sequence available, more transcripts will be examined to

18 facilitate future research on M. sexta fat body, hemocytes and midgut. Our recent study reported

19 miRNA profiles in $M$. sexta fat body and hemocytes with or without pathogen stimulation and

20 suggested potential regulatory pairs of miRNAs and immunity-related genes (Zhang et al., 2014).

21 Sequencing the tissue RNAs yielded better coverage of the miRNA repertoire than the whole

22 body samples (Zhang et al., 2012). However, tissue specificity of miRNA expression has not yet

23 been explored in fat body or hemocytes. Neither is information available on how miRNAs may

24 regulate $M$. sexta metabolic processes or if some miRNAs co-regulate metabolism and immunity. 
Therefore, we attempted to identify more miRNAs, examine tissue-preferential expression of

2 miRNAs and, more importantly, discover possible miRNA coordination of metabolism and

3 systematic immune responses. To achieve these goals, we constructed small RNA libraries of

4 larval fat body, hemocytes and midgut. Each library represented the same tissue in four immune

5 states. For instance, the fat body library was built using a pool of the tissue samples from naïve

6 and induced larvae separately injected with Escherichia coli, Bacillus subtilis, and

7 Saccharomyces cerevisiae. Normalized miRNA abundances were compared and categorized into

8 the three tissue groups. For each group, we identified the transcripts specifically expressed in that

9 tissue of the $5^{\text {th }}$ instar larvae and predicted the target sites in their 3 '-UTRs. The combined

10 analysis of miRNA profiles and mRNA tissue-specificity allowed us to discover potential

11 regulatory relationships at the tissue level in terms of metabolism and immunity. By including

12 midgut tissue samples, pooling tissues in various physiological states, and increasing throughput

13 of sequencing, we obtained an extensive list of conserved and novel miRNAs to complement the

14 M. sexta genome project.

\section{2. Materials and Methods}

\section{2.1. Sample preparation and Illumina sequencing}

18 Three groups of $M$. sexta larvae (day $2,5^{\text {th }}$ instar, six in each group) were injected with heat-

19 killed E. coli $\left(5 \times 10^{7}\right.$ cells/larva), Bacillus subtilis $\left(5 \times 10^{7}\right.$ cells/larva) and Saccharomyces

20 cerevisiae $\left(1 \times 10^{7}\right.$ cells/larva), respectively. After $6 \mathrm{~h}$, hemolymph was collected and centrifuged

21 at $5000 \mathrm{~g}$ for $5 \mathrm{~min}$ to harvest hemocytes. The larvae were subsequently dissected for fat body

22 and midgut tissues. Hemocytes, fat body and midgut tissues were obtained in the same way from

23 six day $3,5^{\text {th }}$ instar naïve larvae. The three tissue pools were separately ground in liquid nitrogen

24 and stored in TRIZOL Reagent (Thermo Fisher Scientific) at $-80^{\circ} \mathrm{C}$ prior to shipping on dry ice. 
1 The small RNA libraries of hemocytes, fat body and midgut were constructed at BGI (Beijing,

2 China) and sequenced using Illumina technology.

\section{2.2. Identification of conserved and novel miRNAs and miRNA profiling}

4 Sequence analyses were performed as described previously (Zhang et al., 2012). Briefly,

5 small RNAs without perfect match to the 3'-adaptor were disposed. Reads were further removed

6 if they resemble repeats, noncoding RNAs (e.g. rRNAs, tRNAs, snRNAs) or mitochondrial

7 RNAs in the respective online databases. Possible mRNA degradation products were filtered out

8 by comparing them with the CIFH dataset (Gunaratna and Jiang, 2013; Zhang et al., 2011; Zou

9 et al., 2008), midgut EST dataset (Pauchet et al., 2010), and Cufflink RNA-Seq Assembly 1.0

10 (http://agripestbase.org/manduca/). Conserved miRNAs were selected according to miRBase

11 (v20, http://www.miRBase.org/). Their loci were obtained by retrieving mature miRNA

12 sequences in $M$. sexta Genome Assembly 1.0 (http://agripestbase.org/manduca/). Corresponding

13 precursor sequences were identified if they had $\geq 18$ matched base pairs, a single central loop,

14 and minimum free energy (MFE) $<-18 \mathrm{kCal} / \mathrm{mol}$ (http://mfold.rit.albany.edu/?q=mfold/RNA-

15 Folding-Form2.3) (Zuker, 2003). Novel miRNA mature sequences were designated based on the

16 following criteria: $\geq 10$ total reads in the three libraries combined, $\leq 5$ genomic loci, precursors

17 identified similarly as the conserved miRNAs, highest abundances compared to other reads

18 mapped to the precursors, and existence of predicted miRNA* strands. The ones without

19 accompanying miRNA* strands are considered to be novel miRNA candidates. Normalized

20 frequencies for both conserved and novel miRNAs were calculated based on the size of each

21 small RNA library. Heat maps of normalized miRNA counts were generated using

22 Multiexperiment Viewer (v4.9) (Saeed et al., 2003). $\log _{2} \mathrm{NC}$ values (NC for normalized count)

23 were actually used as data input. If an $\mathrm{NC}<0.5, \log _{2} 0.5$ was taken to represent the low miRNA

24 abundance. Figure of Merit (FOM) was plotted to determine an appropriate number of K-means 
1 clusters with Pearson correlation. Pearson correlation and K-means clustering with maximum

2 iterations of 50,000 were chosen for creating the heat maps. Color scale limits were set at 0 for

3 low (blue) and at 22.1 for high (red), where 22.1 was the highest $\log _{2} \mathrm{NC}$ value in the dataset.

4 2.3. Detection of tissue-specific transcripts in M. sexta fat body and midgut

5 Specific transcripts were selected based on results from analysis of the 52 RNA-Seq datasets

6 corresponding to different tissues and stages of M. sexta (Cao et al., unpublished data). Briefly,

7 reads from all these datasets were first assembled using Trinity (Grabherr et al., 2011) and

8 FPKMs were calculated by RSEM (Li and Dewey, 2011). Due to sampling differences, the

9 datasets of fat body and midgut from day $1,5^{\text {th }}$ instar larvae were used to calculate transcript

10 levels, detect tissue specific expression, and correlate with miRNA levels, since this stage is the

11 closest to that used for constructing the three small RNA libraries. Ratio for a specific transcript

12 was calculated using its FPKM in the fat body or midgut dataset over its average FPKM in

13 datasets excluding the same tissue. Initial screens for tissue-specific transcripts were performed

14 under the following conditions: transcript length $>300$ nt, FPKM $>15$ (for fat body) and 25 (for

15 midgut $)$, ratio $>3$, and $\log _{10}($ ratio $) \times \log _{10}(\mathrm{FPKM}$ in the two datasets $)>1$. Redundant transcripts

16 were removed if they are $>90 \%$ similar in sequence, their translated protein sequences are $<80$

17 residues, or transcripts have no 3'-UTRs. Proteins encoded by the remaining transcripts were

18 used as queries to perform BLASTP search of uniprotKB_Arthropoda (ftp://ftp.ebi.ac.uk/pub/

19 databases/fastafiles/uniprot/uniprotkb_arthropoda.gz). Transcripts that had no hit or only hit

20 uncharacterized proteins were no longer analyzed.

21 2.4. miRNA target site analysis

22 The fat body- and midgut-specific transcripts were selected for target site prediction, along 23 with the eleven hemocyte-specific transcripts (Zhang et al., 2011). 3'-UTRs were collected for $M$.

24 sexta miRNA target site analysis using Hitsensor (Zheng and Zhang, 2010). 


\section{3. Results}

\section{3.1. Overview of the dataset}

$4 \quad$ From the three small RNA libraries, we in total obtained $\sim 167$ million reads, far exceeding 5 the 21.1 and 32.9 million in our previous datasets (Zhang et al., 2012), (Zhang et al., 2014). BGI

6 proprietary technologies of small RNA library construction and sequencing apparently led to the

7 differences. The previous studies retrieved small RNAs in the range of 18-31 nt, whereas the 8 current one recovered reads of 18-44 nt in length (Fig. 1), in spite of low counts for reads longer

9 than $31 \mathrm{nt}$. The new dataset comprised higher percentages of noncoding RNAs, miRBase 10 precursors, hemocyte-fat body-midgut ESTs, Cufflink transcripts, repeats, and Genome 11 Assembly 1.0 (Table 1). However, in terms of the unique read percentages for each library, they 12 were similar to the dataset of M. sexta CIFH small RNAs (Zhang et al., 2014) in the categories 13 of noncoding RNAs, miRBase precursors, and repeats. Compared with hemocytes, fat body and 14 midgut had higher percentages of unique reads matching hemocyte-fat body-midgut ESTs, 15 Cufflink transcripts, and Genome Assembly 1.0. Since reads were removed if they matched 16 noncoding RNAs, mRNA fragments and repeats, the differences should not adversely affect 17 miRNA identification or profiling. The ratios of total counts over unique counts, representing 18 miRNA average abundances, were 693, 988, and 188 for fat body, hemocytes and midgut, 19 respectively, much higher than those of $\mathrm{CF}$ (56), IF (51), $\mathrm{CH}$ (85) and $\mathrm{IH}$ (79) (Zhang et al., 20 2014).

\section{3.2. Identification of additional M. sexta miRNAs}

22 We have identified another six novel miRNAs, one of which, $\mathrm{t} 4742895$, was present at much

23 higher levels (Table 2). Their corresponding precursors could form canonical stem-loop 24 structures with MFE lower than $-18 \mathrm{kcal} / \mathrm{mol}$ (Fig. 2 and Table 2). t357780, t454580, t2290056 
1 and t3057689 were at low levels and it might be possible that those four miRNAs were not

2 preferentially expressed in the three selected tissues at the specific sampling time. Due to the

3 failure of detecting their corresponding star strands, t357780, t454580, t3057689 and t3301296

4 were reported as novel miRNA candidates (Zhang et al., 2014), while t4742895 was reported as

5 s856646 (Zhang et al., 2012). These five candidates were present at extremely low levels in the

6 old datasets. The most dramatic example was s856646 or t4742895, whose read numbers in the

7 whole body libraries of embryos (20), larvae (9), pupae (11) and adults (32) were much lower

8 than those in the $5^{\text {th }}$ instar larval fat body $(131,975)$, hemocytes $(66,051)$ and midgut $(41,738)$.

9 Due to spatiotemporal specificity of miRNA expression, more candidates are expected to be

10 advanced to novel miRNAs in future small RNA library sequencing projects covering other

11 tissues or time points. Here we present another 26 novel miRNA candidates (Table S1). Among

12 them, small RNAs t6322016 and t3125494 may be derived from two precursors, and the two

13 precursors of t6322016 arose from convergent templates at the same genomic loci, rendered as

14 antisense miRNA candidates. Interestingly, t6322016 matched the predicted precursors of novel

15 miRNA candidates s391130a and s391130b, and those two were reported as antisense miRNA

16 candidates as well (Zhang et al., 2012). Either s391130 or t6322016 might be a genuine miRNA

17 resulting from 301078 to $301178 \mathrm{nt}$ of Scaffold 352 (Table S1). Identification of additional novel

18 miRNAs will facilitate genome annotation, miRNA clustering analysis on genome scaffolds and

19 examination of closely resided genes to various miRNA loci.

20 We previously identified conserved M. sexta miRNAs with or without precursors (Zhang et

21 al., 2012). There are two new conserved miRNA variants without precursors in the current

22 dataset: miR-281 (ACUGUCAUGGAGUUGCUCUCUU) and miR-305 (AUUGUACUUCAUC

23 AGGUGCUCU). We have normalized miRNA reads based on total read numbers of each library

24 (Table 3 and Table S2) and Table 3 also includes novel miRNAs. 


\subsection{Certain miRNA* strands maintained at considerable levels}

In the past, we proposed that certain miRNA star strands present at substantial levels may manipulate target gene expression and that two strands derived from a single miRNA precursor may regulate different targets (Zhang et al., 2014). For example, mse-miR-8* level fluctuates in fat body and hemocytes upon immune challenge and potentially regulates transcripts different from mse-miR- 8 targets. mse-miR- $8 *$ is abundant in the current dataset. We have also found the star strands of mse-miR-9a, -9b, -10a, -14, $-31,-71,-281,-308,-965$ and -6097 were more abundant than their corresponding mature strands (Table 3). Three of them (mse-miR-31*, $-281^{*}$, and $-965^{*}$ ) were reported before (Zhang et al., 2012, 2014). Their precursors may favorably load the star strands into RISC. mse-miR-6097 and $-6097^{*}$, first identified at low levels in the development series (Zhang et al., 2012), were much higher in the three tissues, especially hemocytes. Although mse-miR-9a* and $-9 b^{*}$ were reported at levels similar to the mature strands (Zhang et al., 2012, 2014), it is unclear why mse-miR-9a*, $-9 b^{*},-10 a^{*},-14^{*},-71^{*}$ and $-308^{*}$ were remarkably more abundant in this dataset. It is worth noting that $5 p$ - or $3 p$-arm miRNA dominant usage can be altered by the disease status: some prefer $5 \mathrm{p}$-arms in normal tissue while others prefer 3p-arms in tumor tissue (Li et al., 2012b). However, different bacterial pathogens stimulated similar immune genes in the honeybee (Lourenco et al., 2013). There is no evidence supporting that insect miRNAs differentially react with different killed pathogens. The shorter treatment time and simpler pathogen combination may be related to the fact those six star strands were maintained at substantially higher levels. Differences in library construction procedures may also contribute to the higher abundances of miRNA*s since in the previous $M$. sexta studies small RNA libraries were constructed before they were delivered for sequencing (Zhang et al., 2012, 2014). Therefore, the current dataset might be less consistent with the previous ones.

24 3.4. Abundances of M. sexta miRNAs in fat body, hemocytes and midgut 
To examine expression patterns, we focused on the $M$. sexta miRNAs with identified

2 precursors. After removing those with fewer than 10 normalized reads per million in each tissue,

3 we categorized the remaining 102 miRNAs or miRNA*s into eight clusters based on K-means

4 clustering (Fig. 3). After a closer examination of their normalized abundances, we organized the

5 eight clusters into four groups. The first four clusters (group A) represented the 57 with higher

6 abundances in hemocytes (Fig. 3A) - mse-miR-929b and -279c were also preferentially

7 expressed in fat body. The next two clusters (group B) contained 27 entries preferentially

8 expressed in fat body. mse-miR-100, -279b, -279d, -306 and t4742895* also exhibited high

9 abundances in hemocytes; mse-miR-277 and $-9 \mathrm{a} *$ showed expression preference in midgut as

10 well. One cluster (group C) represented the nine preferentially expressed in midgut except mse-

11 miR-2755* (Fig. 3C). The last cluster (group D) had seven highly expressed in both fat body and

12 midgut (Fig. 3D) and two (mse-miR-283 and -283*) preferentially expressed in midgut only

13 (Table 3). In summary, 36, 62 and 19 of the 102 miRNAs or miRNA*s were preferentially

14 expressed in fat body, hemocytes and midgut, respectively.

15 3.5. miRNAs potentially targeting tissue-specific transcripts in fat body, hemocytes and midgut

16 Even though the preferential miRNA $(*)$ groups were established based only on three tissues,

17 that does not exclude their possible regulatory roles in specific tissues, especially when they are

18 highly abundant. Therefore, it is intriguing to investigate what genes might be regulated in those

19 tissues. From RNA-Seq data of 52 libraries of brain, fat body, midgut, muscle, Malpighian

20 tubules, testes and ovary at various developmental stages, we identified 284 and 485 transcripts

21 specifically expressed in day $1,5^{\text {th }}$ instar larval fat body and midgut, respectively. Because

22 hemocytes were not included in those 52 libraries, we selected and improved eleven hemocyte-

23 specific transcripts based on the result from a previous transcriptome study (Zhang et al., 2011).

24 Since most miRNAs recognize target mRNAs in the 3'-UTR, we used these regions from the 
1 tissue-specific transcripts to predict target sites of the preferential miRNAs in the corresponding

2 tissues.

3 In the 3' UTRs of hemocyte-specific transcripts for aminoacylase, hemolectin, HP1, IML-3a,

4 IML-3b, lacunin, lectin, proPO-1, proPO-2, serpin 2 and SR-C like protein, we searched putative

5 binding sites of the 62 miRNAs preferentially expressed in hemocytes. mse-miR-11*, t3301296,

6 t3301296 and mse-miR-6093* potentially target aminoacylase, IML-3a, lacunin and serpin 2

7 transcripts, respectively (Table 4). Lectin and proPO-1 transcripts contained recognition sites of

8 several miRNAs. MicroRNAs mse-miR-6093*, -6096-1, -6096-2, -6096-3 and t3301296 were

9 identified as novel miRNAs (Zhang et al., 2012). t3301296 potentially regulates both IML-3a

10 and lacunin, two arms of the mse-miR-11 precursor may regulate different targets (Table 4), and

11 mse-miR-11, -190 and -308 may regulate proPO-1 (Zhang et al., 2014). While mse-miR-308 was

12 preferentially expressed in fat body, we excluded mse-miR-bantam* because of its extreme

13 scarcity. Thus, by examining miRNA levels and tissue specificity, we can narrow down putative

14 target lists and provide guidelines for functional verification.

15 Using 166 fat body- and 174 midgut-specific transcripts along with the 36 and 19 preferential

16 miRNAs, we predicted recognition sites in the 3'-UTRs. Among the 166 transcripts in fat body,

1762 may be involved in carbohydrate, lipid, amino acid, or xenobiotic metabolism and 19 in

18 innate immunity (Table 5). Similarly, 82 of the 174 transcripts in midgut, involved in the

19 aforementioned metabolism, are possibly regulated by the 19 miRNAs preferentially expressed

20 in the same tissue (Table 6).

\section{4. Discussion}

\section{4.1. Potential miRNA regulation of metabolic processes}

24 After further examination of the fat body- and midgut-specific transcripts, we found the ones 
1 encoding proteins involved in electron transport and metabolism of carbohydrates, lipids,

2 hormones, amino acids, and xenobiotics. Except for mse-miR-281*, $-92 b^{*}$, and -279b, all 36 fat

3 body- and 19 midgut-preferential miRNAs had at least one possible target for these physiological

4 processes. In the following discussion, we included potential regulatory miRNAs in the

5 parentheses next to the transcript names.

\section{$6 \quad$ 4.1.1. Carbohydrate metabolism}

7 There were five fat body and three midgut transcripts involved in carbohydrate degradation 8 or synthesis. Brain chitinase and chia (mse-miR-79) and $\alpha$-amylase (mse-miR-283) may 9 hydrolyze the polysaccharides. Phosphonenolpyruvate synthase (mse-miR-316, $-9571 \mathrm{a},-281^{*}$ ) is

10 a member of the glycolytic pathway. Trehalose, a major carbohydrate in insect hemolymph, is

11 moved into cells by trehalose transporter Tret1 (mse-miR-2763, -iab-4). Glycogen synthase

12 (mse-miR-10a, -12, -252, -2763, - - *), trehalose-6-phosphate synthase (mse-miR-1, - $8^{*}$ ), and

13 UDP-glucose pyrophosphatase (mse-miR-263b, -277, -306, -2763, -2768) in fat body and

14 mannose-1-phosphate-guanylyltransferase (mse-miR-1) in midgut synthesize carbohydrates.

15 Interestingly, mse-miR-1 and $-8 *$ may each regulate two transcripts. Meanwhile, mse-miR-2763

16 had recognition sites on 3'-UTRs of Tret1, glycogen synthase and UDP-glucose pyrophosphatase,

17 suggestive of a role in trehalose uptake for storage in the form of glycogen.

18 Pentose phosphate pathway is crucial for the carbohydrate conversion. One midgut- and eight

19 fat body-specific transcripts encode enzymes either in the pathway or providing pathway

20 intermediates. They are: 6-phosphogluconate dehydrogenase (mse-miR-1, -282), 6-phosphoglu-

21 conolactonase (mse-miR-282, t4742895), fructose-biphosphate aldolase (mse-miR-2763), phos-

22 phorglucomutase (mse-miR-10a*), phosphoribosyl pyrophosphate synthetase (mse-miR-1),

23 transaldolase (mse-miR-277, -2763), L-xylulose reductase (mse-miR-9a*, -9b*, t4742895*),

24 galactokinase-like protein (mse-miR- $8^{*}$ ), and glucose dehydrogenase (mse-miR-137, -263b, - 
$\left.19 a^{*}\right)$. mse-miR-1, $-282,-2763$ and $-9 a^{*}$ could each regulate two transcripts for the pathway

2 members. Moreover, mse-miR-1, $-277,-263 \mathrm{~b},-2763$ and $-8^{*}$, as potential regulators of

3 carbohydrate conversion, may target genes in carbohydrate synthesis

$4 \quad$ 4.1.2. Lipid metabolism

5 We found fat body-specific transcripts coding for proteins involved in fatty acid synthesis 6 and lipid transport. Acetyl-CoA carboxylase (mse-miR-263b, -iab-4), ATP citrate lyase (mse-

7 miR-10a, -277, -989), elongase (mse-miR-12, -79, -2763, -2768, -let-7a, $-8 *$ ), fatty acid synthase

8 (mse-miR-92b, -2763), long chain fatty acid elongase (mse-miR-1, -92a, -277, -281, -308), acyl-

9 CoA $\Delta 9$ desaturase (m.106622) (mse-miR-2768), and acyl-CoA $\Delta 9$ desaturase (m.46170) (mse-

10 miR-263a) participate in fatty acid synthesis. mse-miR-277, -2763 , and -2768 may each regulate

11 two targets. Acetyl-CoA carboxylase catalyzes the rate-limiting step of fatty acid synthesis and,

12 thus, mse-miR-263b and -iab-4 may contribute to the rate modulation. Transcripts of fatty acid

13 synthase contain recognition sites of mse-miR-92b and -2763 in the 3'-UTR. Awaiting future

14 analysis is which one(s) of the four miRNAs (92b, 263b, iab-4 and 2763) are important to control

15 fatty acid synthesis. Fat body is the main storage site for acylglycerols; adipokinetic hormone

16 (AKH) induces the conversion of triacylglycerols to diacylglycerols for transportation to other

17 tissues. Fat body-specific transcripts for the AKH receptor (mse-miR-263a, -iab-4, - $8^{*}$ ) and its

18 potential regulatory miRNAs should be tested for AKH binding. We also found two transcripts

19 in fat body for lipid transport, apolipophorins (mse-miR-308) and apolipoprotein of lipid transfer

20 particle III (mse-miR-2763, -2768).

21 In contrast, midgut-specific transcripts are responsible for acylglycerol hydrolysis and $\beta$ -

22 oxidation. Lipase (mse-miR-1), LPP1 (mse-miR-283), pancreatic lipase (mse-miR-137, -6094)

23 and lysosomal acid lipase (mse-miR-263b, -9571b) take part in acylglycerol hydrolysis; acyl-

24 CoA dehydrogenase (mse-miR-263a), enoyl-CoA hydratase (mse-miR-137) and enoyl-CoA 
1 isomerase (mse-miR-263a) in fatty acid $\beta$-oxidation. Since mse-miR-263a potentially regulates

2 AKH receptor, acyl-CoA dehydrogenase, and enoyl-CoA isomerase, it is possible that this 3 miRNA plays a significant role in fatty acid catabolism.

$4 \quad$ 4.1.3. TCA cycle and electron transport chain and ATPases

5 TCA cycle is crucial for energy production and intermediate conversion. One fat body- and

6 four midgut-specific transcripts are found: malate dehydrogenase (mse-miR-1, -263a, -278), 7 citrate synthase (mse-miR-9a*), isocitrate dehydrogenase (m. 30761) (mse-miR-137, -277, 8 9571b), isocitrate dehydrogenase (m. 61421) (mse-miR-283), and succinyl-CoA ligase $\beta$ chain 9 (mse-miR-316). Among them, isocitrate dehydrogenase is important to regulate the whole TCA 10 cycle and, therefore, it is interesting to test which one of the two transcripts encodes TCA 11 isocitrate dehydrogenase and what miRNAs may regulate it. Reducing equivalents are needed for 12 the respiratory chain to cause ATP synthesis while the malate-aspartate shuttle can transport 13 cytosolic NADH into mitochondria. The midgut-specific transcripts of cytosolic malate 14 dehydrogenase (mse-miR-137, -283), together with malate dehydrogenase, is an important 15 component of the shuttle. The two enzymes may be regulated by different miRNAs in terms of 16 enzyme expression levels. Cytochrome b5 (mse-miR-10a, -281, -316) in both fat body and 17 midgut and cytochrome c homolog (mse-miR-281*) in midgut are members of the electron 18 transport chain. There were 9 midgut transcripts encoding for ATP synthase/ATPases, but those 19 were the vacuolar type which is not associated with the mitochondrial ATPases. Nevertheless, it 20 is intriguing that mse-miR-1 had predicted target sites in seven 3'-UTRs of those nine transcripts.

21 Recent studies found that although miRNAs are encoded by nuclear genes, certain miRNAs can

22 accumulate in mammalian mitochondria and components of miRNA machinery were detected

23 there ( $\mathrm{Li}$ et al., 2012a). While there is no evidence that insect miRNAs also accumulate in 24 mitochondria to regulate mitochondrial activity, we hypothesize insect mitochondria have 
1 miRNA regulatory machinery as well. We found four fat body- and seven midgut-specific

2 transcripts encoding mitochondrial proteins potentially regulated by miRNAs (Table 7). It would

3 be interesting to verify which miRNAs are enriched in insect mitochondria to associate with their

4 targets.

5 4.1.4. Amino acid metabolism

6 We found fat body-specific transcripts of amino acid synthases and other enzymes for Gly,

7 Ser, Thr and Met metabolism, including Asn synthase (mse-miR-8, -282, -2763, t4742895*), Cys

8 synthase (mse-miR-277), Gly cleavage system h protein (mse-miR-92b), Gly cleavage system $\mathrm{p}$

9 protein (mse-miR-12), Asn tRNA synthetase (mse-miR-277), D-3-phosphoglycerate

10 dehydrogenase (mse-miR-92b, -308), Gly $N$-methyltransferase (mse-miR-12, -92b, -277 and -

11 282), phosphatidylserine decarboxylase (mse-miR-308) and phosphoserine phosphatase (mse-

12 miR-277). D-3-phosphoglycerate hydrogenase is the rate limiting step for Ser synthesis. mse-

13 miR-92b and 308 may regulate three (D-3-phosphoglycerate dehydrogenase, glycine cleavage

14 system h, and glycine N-methyltransferase) and two (D-3-phosphoglycerate hydrogenase and

15 phosphatidylserine decarboxylase) in glycerine, serine and threnoine metabolic processes,

16 respectively. mse-miR-12 and 277 were two other interesting candidates because they each

17 potentially regulate two transcripts in Gly, Ser and Thr metabolism. For Met metabolism, there

18 were two transcripts encoding hemocysteine-S-methyltransferase, m. 156992 (mse-miR-iab-4, -

$199 \mathrm{a}^{*},-9 \mathrm{~b}^{*}$ ) and m.78459 (mse-miR-2763).

20 We found a fat body transcript, kynurenine formamidase (mse-miR-let-7a), and a midgut 21 transcript, kynureninase (mse-miR-283, -316, -9571b), for tryptophan metabolism. Two midgut

22 transcripts, isovaleryl-CoA dehydrogenase (mse-miR-10a and $-316^{*}$ ) and methylcrotonoyl-CoA

23 carbohylase $1 \alpha$ (mse-miR-10a* and -750*), are involved in Leu, Ile and Val metabolism. The 5p-

24 or 3p-arm of mse-miR-10a precursor may regulate one of the two transcripts, implying that mse- 
1 miR-10a gene is important for Leu, Ile and Val metabolism. The conversion of amino groups

2 into uric acid is important for disposal of excessive nitrogen in insects and there were four

3 transcripts involved, branched-chain amino acid aminotransferase (mse-miR-33, -929, -2763, -

42768 and $-9 a^{*}$ ), nitrilase (mse-miR-263a), amidase (mse-miR-263b), and aminoacyalase-1 (mse-

$5 \quad \operatorname{miR}-283)$.

\section{$6 \quad$ 4.1.5. Xenobiotic metabolism}

7 There were five transcripts for cytochrome P450s and four transcripts for glutathione S8 transferases, which are significant for xenobiotic metabolism in insects. mse-miR-9571b, a novel 9 miRNA, may regulate three glutathione S-transferase transcripts; mse-miR-316* could recognize

10 two glutathione S-transferases and one cytochrome P450. We also found aldehyde oxidase-2 11 (mse-miR-10a*) for cytochrome P450 oxidation and three enzymes involved in glutathione 12 metabolism, $\gamma$-glutamyl cyclotransferase like (mse-miR- $8^{*}$ ), $\gamma$-glutamyltransferase (mse-miR$\left.132763,-278^{*}\right)$ and lactoylglutathione lyase (mse-miR-9a*, $\left.-9 b^{*},-750^{*}\right)$. RNA interference has 14 recently been utilized to affect expressions of cytochrome P450s, esterases or cadherin proteins 15 to reduce insecticide resistance (Tang et al., 2010). Upon validation, this knowledge might be 16 used to affect insecticide resistance via specific miRNAs. Last but not the least, one fat body 17 transcript for $\mathrm{ABC}$ transporter ( $\mathrm{t} 4742895)$ and one midgut transcript amino-peptidase N-11 (mse18 miR-283) are potentially involved in the resistance to Bt toxin (Gahan et al., 2010;

19 Likitvivatanavong et al., 2011).

20 4.1.6. Hormonal control

21 Ecdysteriods and juvenile hormones (JHs) are important lipid hormones to regulate various

22 physiological processes in insects. We identified two fat body transcripts, JH epoxide hydrolase 23 (mse-miR-100) and JH esterase (mse-miR-92a, -92b, -277, -929 and -let-7a) for JH inactivation.

24 We also found a fat body transcript for ecdysone oxidase (mse-miR-12) and a midgut transcript 
1 for 3-hydroecdysone-3 $\alpha$-reductase (mse-miR-281, -316 and -283*) involved in ecdysone

2 inactivation. Binding proteins of hemolymph JH (mse-miR-33, -2763, -2768) and cytosolic JH

3 (mse-miR-9571a) were preferentially expressed in fat body and midgut, respectively. Since JH is

4 not synthesized in midgut, it is interesting that one midgut specific transcript encodes FAMeT

5 (mse-miR-283). The seven aforementioned transcripts related to hormonal control are possibly

6 regulated by different miRNAs which manipulate the target gene expression.

7 4.2. Possible correlation between immunity and metabolism

8 By examining potential regulatory pairs of tissue-preferential miRNAs and -specific 9 transcripts, we found miRNAs that may regulate genes for metabolic enzymes. This might

10 explain why miRNAs are dysregulated in response to environmental stresses such as freezing

11 and starvation, when hypo-metabolism happens. In T. castanuem, levels of five miRNAs arose

12 while those of 22 miRNAs decreased upon starvation. Out of those 27 changed, we found seven

13 conserved miRNAs (miR-1, $-8,-79,-100,-279 \mathrm{c},-279 \mathrm{~d}$ and -let-7a) could target metabolic genes.

14 These miRNAs were down-regulated in T. castanuem after starvation. Meanwhile, out of the 33

15 fat body preferential miRNAs targeting metabolic gene expression, levels of 18 (mse-miR-1, -8 ,

$16-10 a,-12,-33,-100,-252,-263 a,-279 c,-279 d,-282,-306,-308,-989,-2763$, -let-7a, $\left.-8 *,-9 b^{*}\right)$

17 decreased and levels of 3 (mse-miR-92b, -277 and -iab-4) increased in M. sexta fat body after the

18 immune challenge (Zhang et al., 2014). Thus these 21 miRNAs may correlate metabolism and

19 systematic immune responses.

\section{5. Conclusions}

22 In this finale of our tripartite series, deep sequencing of the small RNAs from tissues under 23 different conditions yielded a more extensive list of M. sexta miRNAs. Comparison of the 24 normalized reads revealed miRNAs preferentially expressed in the tissues. Tethered with tissue- 
1 specific transcripts in fat body and midgut, we identified putative regulatory pairs of miRNAs

2 and transcripts of metabolic enzymes. The initial data and premises help us to rationalize why

3 miRNAs are dysregulated under stresses and how they may co-regulate metabolism and

4 immunity. Along with the development and immunity profiles, we have analyzed experimental

5 data to uncover a miRNA repertoire in the genome of this model insect, which fluctuates in

6 tissues of different immune states at several life stages. After validating their targets in future

7 studies, we can better appreciate the regulatory roles of miRNA in various insect physiological

8 processes.

\section{Acknowledgments}

The research was supported by National Institutes of Health Grant GM58634 (to HJ), and a start-up fund from Kunming University of Science and Technology (to YZ). Part of the computation for this project was performed at OSU High Performance Computing Center at Oklahoma State University supported in part through the National Science Foundation grant OCI-1126330. This article was approved for publication by the Director of the Oklahoma Agricultural Experiment Station and supported in part under projects OKLO2450 (to HJ). We also thank Manduca Genome Project for Genome Assembly 1.0, funded by Defense Advanced Research Projects Agency (Gary Blissard, Boyce Thompson Institute) and National Institutes of Health (Michael Kanost, Kansas State University).

\section{References}

Asgari, S., 2011. Role of MicroRNAs in Insect Host-Microorganism Interactions. Front Physiol 2,48 .

Baker, K.D., Thummel, C.S., 2007. Diabetic larvae and obese flies-emerging studies of metabolism in Drosophila. Cell Metab 6, 257-266.

Chawla, G., Sokol, N.S., 2011. MicroRNAs in Drosophila development. Int Rev Cell Mol Biol 286, 1-65.

Choi, I.K., Hyun, S., 2012. Conserved microRNA miR-8 in fat body regulates innate immune homeostasis in Drosophila. Dev Comp Immunol 37, 50-54. 
Courteau, L.A., Storey, K.B., Morin, P., Jr., 2012. Differential expression of microRNA species in a freeze tolerant insect, Eurosta solidaginis. Cryobiology 65, 210-214.

Davalos, A., Goedeke, L., Smibert, P., Ramirez, C.M., Warrier, N.P., Andreo, U., Cirera-Salinas, D., Rayner, K., Suresh, U., Pastor-Pareja, J.C., Esplugues, E., Fisher, E.A., Penalva, L.O., Moore, K.J., Suarez, Y., Lai, E.C., Fernandez-Hernando, C., 2011. miR-33a/b contribute to the regulation of fatty acid metabolism and insulin signaling. Proc Natl Acad Sci U S A 108, 92329237.

Etebari, K., Asgari, S., 2013. Conserved microRNA miR-8 blocks activation of the Toll pathway by upregulating Serpin 27 transcripts. RNA Biol 10, 1356-1364.

Etebari, K., Hussain, M., Asgari, S., 2013. Identification of microRNAs from Plutella xylostella larvae associated with parasitization by Diadegma semiclausum. Insect Biochem Mol Biol 43, 309-318.

Freitak, D., Knorr, E., Vogel, H., Vilcinskas, A., 2012. Gender- and stressor-specific microRNA expression in Tribolium castaneum. Biol Lett 8, 860-863.

Gahan, L.J., Pauchet, Y., Vogel, H., Heckel, D.G., 2010. An ABC Transporter Mutation Is Correlated with Insect Resistance to Bacillus thuringiensis Cry1Ac Toxin. Plos Genet 6.

Grabherr, M.G., Haas, B.J., Yassour, M., Levin, J.Z., Thompson, D.A., Amit, I., Adiconis, X., Fan, L., Raychowdhury, R., Zeng, Q.D., Chen, Z.H., Mauceli, E., Hacohen, N., Gnirke, A., Rhind, N., di Palma, F., Birren, B.W., Nusbaum, C., Lindblad-Toh, K., Friedman, N., Regev, A., 2011. Full-length transcriptome assembly from RNA-Seq data without a reference genome. Nat Biotechnol 29, 644-U130.

Gunaratna, R.T., Jiang, H., 2013. A comprehensive analysis of the Manduca sexta immunotranscriptome. Dev Comp Immunol 39, 388-398.

Hakimi, M.A., Cannella, D., 2011. Apicomplexan parasites and subversion of the host cell microRNA pathway. Trends Parasitol 27, 481-486.

Hiruma, K., Riddiford, L.M., 2010. Developmental expression of mRNAs for epidermal and fat body proteins and hormonally regulated transcription factors in the tobacco hornworm, Manduca sexta. J Insect Physiol 56, 1390-1395.

Hyun, S., Lee, J.H., Jin, H., Nam, J., Namkoong, B., Lee, G., Chung, J., Kim, V.N., 2009. Conserved MicroRNA miR-8/miR-200 and its target USH/FOG2 control growth by regulating PI3K. Cell 139, 1096-1108.

Li, B., Dewey, C.N., 2011. RSEM: accurate transcript quantification from RNA-Seq data with or without a reference genome. Bmc Bioinformatics 12.

Li, P., Jiao, J., Gao, G., Prabhakar, B.S., 2012a. Control of mitochondrial activity by miRNAs. J Cell Biochem 113, 1104-1110.

Li, S.C., Liao, Y.L., Ho, M.R., Tsai, K.W., Lai, C.H., Lin, W.C., 2012b. miRNA arm selection and isomiR distribution in gastric cancer. Bmc Genomics 13.

Likitvivatanavong, S., Chen, J.W., Bravo, A., Soberon, M., Gill, S.S., 2011. Cadherin, Alkaline Phosphatase, and Aminopeptidase $\mathrm{N}$ as Receptors of Cry11Ba Toxin from Bacillus thuringiensis subsp. jegathesan in Aedes aegypti. Appl Environ Microb 77, 24-31.

Lourenco, A.P., Guidugli-Lazzarini, K.R., Freitas, F.C.P., Bitondi, M.M.G., Simoes, Z.L.P., 2013. Bacterial infection activates the immune system response and dysregulates microRNA expression in honey bees. Insect Biochem Molec 43, 474-482.

Lyons, P.J., Lang-Ouellette, D., Morin, P., Jr., 2013a. CryomiRs: towards the identification of a cold-associated family of microRNAs. Comp Biochem Physiol Part D Genomics Proteomics 8, 358-364.

Lyons, P.J., Poitras, J.J., Courteau, L.A., Storey, K.B., Morin, P., Jr., 2013b. Identification of 
differentially regulated micrornas in cold-hardy insects. Cryo Letters 34, 83-89.

Marco, A., Hui, J.H., Ronshaugen, M., Griffiths-Jones, S., 2010. Functional shifts in insect microRNA evolution. Genome Biol Evol 2, 686-696.

Pauchet, Y., Wilkinson, P., Vogel, H., Nelson, D.R., Reynolds, S.E., Heckel, D.G., ffrenchConstant, R.H., 2010. Pyrosequencing the Manduca sexta larval midgut transcriptome: messages for digestion, detoxification and defence. Insect Mol Biol 19, 61-75.

Saeed, A.I., Sharov, V., White, J., Li, J., Liang, W., Bhagabati, N., Braisted, J., Klapa, M., Currier, T., Thiagarajan, M., Sturn, A., Snuffin, M., Rezantsev, A., Popov, D., Ryltsov, A., Kostukovich, E., Borisovsky, I., Liu, Z., Vinsavich, A., Trush, V., Quackenbush, J., 2003. TM4: a free, open-source system for microarray data management and analysis. Biotechniques 34, 374-378.

Stanley, D., Miller, J., Tunaz, H., 2009. Eicosanoid actions in insect immunity. J Innate Immun 1, 282-290.

Tang, T., Liu, X., Qiu, L., 2010. RNA interference and its applications on silencing of insecticide-resistant genes in insects. Cotton Science 22, 617-624.

Zhang, S., Gunaratna, R.T., Zhang, X., Najar, F., Wang, Y., Roe, B., Jiang, H., 2011. Pyrosequencing-based expression profiling and identification of differentially regulated genes from Manduca sexta, a lepidopteran model insect. Insect Biochem Mol Biol 41, 733-746.

Zhang, X., Zheng, Y., Jagadeeswaran, G., Ren, R., Sunkar, R., Jiang, H., 2012. Identification and developmental profiling of conserved and novel microRNAs in Manduca sexta. Insect Biochem Mol Biol 42, 381-395.

Zhang, X., Zheng, Y., Jagadeeswaran, G., Ren, R., Sunkar, R., Jiang, H., 2014. Identification of conserved and novel microRNAs in Manduca sexta and their possible roles in the expression regulation of immunity-related genes. Insect Biochem Mol Biol 47, 12-22.

Zheng, Y., Zhang, W., 2010. Animal microRNA target prediction using diverse sequencespecific determinants. Journal of Bioinformatics and Computational Biology 8, 763-788.

Zou, Z., Najar, F., Wang, Y., Roe, B., Jiang, H., 2008. Pyrosequence analysis of expressed sequence tags for Manduca sexta hemolymph proteins involved in immune responses. Insect Biochem Mol Biol 38, 677-682.

Zuker, M., 2003. Mfold web server for nucleic acid folding and hybridization prediction. Nucleic Acids Res 31, 3406-3415.

Table 1. Absolute read counts for different RNA categories*

\begin{tabular}{c|rr|rr|rr}
\hline \multirow{2}{*}{ Category } & \multicolumn{2}{|c|}{ fat body } & \multicolumn{2}{c}{ hemocytes } & \multicolumn{2}{c}{ midgut } \\
\cline { 2 - 7 } & \multicolumn{1}{|c|}{ total } & unique & \multicolumn{1}{c}{ total } & unique & \multicolumn{1}{c}{ total } & unique \\
\hline noncoding RNAs & $39,884,461$ & 318,413 & $42,756,639$ & 424,712 & $43,986,901$ & 564,265 \\
miRBase precursors & $14,426,796$ & 20,816 & $33,224,513$ & 33,632 & $9,748,026$ & 51,797 \\
hemocyte, fat body \& midgut & $33,187,609$ & $1,144,452$ & $6,976,905$ & 321,113 & $40,613,282$ & $1,285,436$ \\
Cufflink transcripts & $26,649,634$ & $1,420,730$ & $35,688,300$ & 519,407 & $21,060,022$ & $1,321,070$ \\
Repeats & $8,405,172$ & 103,019 & $4,519,342$ & 139,428 & $12,297,243$ & 196,431 \\
Genome Assembly 1.0 & $40,295,140$ & $1,766,651$ & $42,846,338$ & 786,176 & $35,183,741$ & $1,606,036$ \\
Total & $56,131,508$ & $2,601,196$ & $54,574,527$ & $1,930,668$ & $56,372,683$ & $2,479,566$ \\
\hline
\end{tabular}

* Unique read numbers are ones after the removal of redundant reads.

Table 2. Newly discovered $M$. sexta novel miRNAs*

\begin{tabular}{|c|c|c|c|c|c|c|c|c|}
\hline \multirow{2}{*}{ name } & \multirow{2}{*}{ mature miRNA sequence } & \multicolumn{2}{|c|}{ fat body } & \multicolumn{2}{|c|}{ hemocytes } & \multicolumn{2}{|c|}{ midgut } & \multirow{2}{*}{$\begin{array}{l}\text { precursor } \\
\text { MFE }\end{array}$} \\
\hline & & $\mathrm{miR}$ & $\mathrm{miR}^{*}$ & $\mathrm{miR}$ & $\mathrm{miR}^{*}$ & $\mathrm{miR}$ & $\mathrm{miR}^{*}$ & \\
\hline t357780 & AAGGCGGUAAUUGUUGUUGAG & 23 & 4 & 1 & 1 & 8 & 2 & -30.2 \\
\hline $\mathrm{t} 454580$ & AAUCGGACAAGCAGUGCGAACG & 0 & 1 & 10 & 2 & 3 & 0 & -34.8 \\
\hline t2290056 & CGACCCUUGACAAAUCUUCUGA & 3 & 4 & 12 & 4 & 5 & 4 & -39.6 \\
\hline
\end{tabular}




\begin{tabular}{ll|cc|cc|cc|c} 
t3057689 & GACAGAGAUUGUGGCAAGUCGUC & 2 & 2 & 7 & 8 & 1 & 0 & -29.1 \\
t3301296 & GAUGUACUGACUGUUUCAUCGC & 75 & 0 & 598 & 2 & 147 & 0 & -35.7 \\
t4742895 & UAUUAGAGUUCUGUGCUUCCUG & 131975 & 157 & 66051 & 113 & 41738 & 47 & -32.7 \\
\hline
\end{tabular}

${ }^{*}$ Read numbers are absolute values from each library. The unit of precursor MFE is $\mathrm{kcal} / \mathrm{mol}$.

Table 3. Abundances of 71 conserved and 16 novel miRNAs with precursors identified*

\begin{tabular}{|c|c|c|c|c|c|c|}
\hline \multirow{2}{*}{ Name } & \multicolumn{3}{|c|}{ miRNA } & \multicolumn{3}{|c|}{ miRNA* $^{*}$} \\
\hline & fat body & hemocytes & midgut & fat body & hemocytes & midgut \\
\hline mse-miR-1 & 307471 & 1976 & 392494 & & & \\
\hline mse-miR-1b & 3 & 7 & 4 & & & \\
\hline mse-miR-2a & 266 & 468 & 131 & 75 & 228 & 42 \\
\hline mse-miR-2b & 229 & 400 & 113 & 71 & 113 & 16 \\
\hline mse-miR-7 & 525 & 3786 & 466 & 1 & 1 & 1 \\
\hline mse-miR-8 & 244823 & 25317 & 9301 & 59496 & 20768 & 3579 \\
\hline mse-miR-9a & 128 & 182 & 27 & 425741 & 209874 & 356777 \\
\hline mse-miR-9b & 6 & & 1 & 16233 & 191 & 4575 \\
\hline mse-miR-10a & 942 & 64 & 1401 & 2982 & 152 & 3372 \\
\hline mse-miR-10b & & 3 & & & & \\
\hline mse-miR-10c & & 3 & & & & \\
\hline mse-miR-11 & 1656 & 4572 & 873 & 58 & 218 & 47 \\
\hline mse-miR-12 & 11130 & 241 & 4453 & & & \\
\hline mse-miR-14 & 949 & 1787 & 665 & 709 & 2574 & 1099 \\
\hline mse-miR-31 & 2 & 2 & 2 & 2119 & 4558 & 3488 \\
\hline mse-miR-33 & 29 & 8 & 11 & 2 & 5 & 1 \\
\hline mse-miR-34 & 33 & 85 & 38 & 7 & 7 & 2 \\
\hline mse-miR-71 & 142 & 212 & 43 & 1731 & 3427 & 329 \\
\hline mse-miR-79 & 614 & 445 & 202 & 16 & 62 & 13 \\
\hline mse-miR-87 & 265 & 1502 & 767 & 4 & 13 & 2 \\
\hline mse-miR-92a & 14 & 9 & 5 & & & \\
\hline mse-miR-92b & 81 & 33 & 23 & 15 & 9 & 4 \\
\hline mse-miR-100 & 19743 & 13857 & 7546 & & & \\
\hline mse-miR-133 & 3 & & 7 & & & \\
\hline mse-miR-137 & 2 & & 70 & & & \\
\hline mse-miR-184 & 542406 & 4582805 & 311394 & 2 & 51 & 1 \\
\hline mse-miR-190 & 114 & 251 & 59 & 8 & 34 & 9 \\
\hline mse-miR-252 & 4830 & 1569 & 63 & & & \\
\hline mse-miR-263a & 100055 & 9886 & 89284 & 2 & 1 & 4 \\
\hline mse-miR-263b & 1262 & 447 & 1333 & 1 & & 1 \\
\hline mse-miR-275 & 5880 & 7343 & 4927 & 1 & 6 & 2 \\
\hline mse-miR-276 & 16839 & 59742 & 23606 & 20 & 47 & 22 \\
\hline mse-miR-277 & 131 & 47 & 92 & 5 & 3 & 4 \\
\hline mse-miR-278 & 5731 & 1561 & 2541 & 1460 & 922 & 1176 \\
\hline mse-miR-279a & 177 & 456 & 192 & 1 & & 1 \\
\hline mse-miR-279b & 104854 & 81110 & 40043 & 13 & 21 & 10 \\
\hline mse-miR-279c & 1239 & 1431 & 346 & 13 & 21 & 9 \\
\hline mse-miR-279d & 7474 & 5666 & 2880 & 5 & 7 & \\
\hline mse-miR-281 & 2228 & 1 & 1910 & 61841 & 41 & 79371 \\
\hline mse-miR-282 & 240 & 18 & 53 & 9 & 1 & 2 \\
\hline $\begin{array}{l}\text { mse-miR-283 } \\
\text { mse-miR-285 }\end{array}$ & 4314 & $\begin{array}{c}473 \\
1\end{array}$ & 11891 & 63 & 5 & 171 \\
\hline mse-miR-306 & 16657 & 12512 & 5589 & & & \\
\hline mse-miR-307 & 374 & 9890 & 75 & 1 & 60 & \\
\hline mse-miR-308 & 263 & 102 & 52 & 163 & 896 & 139 \\
\hline mse-miR-316 & 18 & & 309 & 1 & & 11 \\
\hline mse-miR-317 & 8454 & 10567 & 6485 & 3 & 3 & 1 \\
\hline mse-miR-745 & 137 & 5127 & 16 & & & \\
\hline mse-miR-750 & 46 & 26 & 6192 & 12 & 6 & 648 \\
\hline mse-miR-929 & & 1 & 1 & & & \\
\hline mse-miR-929b & 18 & 18 & 5 & 1 & & \\
\hline mse-miR-932 & 2 & 18 & 1 & 2 & 19 & \\
\hline mse-miR-965 & 22 & 56 & 5 & 4803 & 10406 & 6723 \\
\hline mse-miR-970 & 460 & 13584 & 127 & 2 & 12 & 1 \\
\hline mse-miR-981-1 & 1 & & 2 & & & \\
\hline mse-miR-981-2 & 1 & & 2 & & & \\
\hline mse-miR-989 & 45 & 12 & 6 & & & \\
\hline mse-miR-993 & 1 & & & & & \\
\hline mse-miR-998 & 105 & 520 & 100 & & & \\
\hline mse-miR-2755 & 411 & 558 & 275 & 11 & 11 & 13 \\
\hline $\begin{array}{l}\text { mse-miR-2763 } \\
\text { mse-miR-2765 }\end{array}$ & $\begin{array}{c}433 \\
6\end{array}$ & $\begin{array}{c}30 \\
3\end{array}$ & $\begin{array}{l}1 \\
3\end{array}$ & & & \\
\hline mse-miR-2766 & 188347 & 321433 & 159054 & 868 & 4978 & 1222 \\
\hline mse-miR-2767 & 994 & 2413 & 32 & & & \\
\hline
\end{tabular}




\begin{tabular}{|c|c|c|c|c|c|c|}
\hline $\begin{array}{l}\text { mse-miR-2768 } \\
\text { mse-miR-2796 } \\
\text { mse-miR-3286 } \\
\text { mse-miR-3338 } \\
\text { mse-miR-6093 } \\
\text { mse-miR-6094 }\end{array}$ & $\begin{array}{c}1 \\
2 \\
200 \\
8653\end{array}$ & $\begin{array}{l}419 \\
145\end{array}$ & $\begin{array}{c}61 \\
228818\end{array}$ & 2 & 58 & 2 \\
\hline $\begin{array}{l}\text { mse-miR-6096-1 } \\
\text { mse-miR-6096-2 } \\
\text { mse-miR-6096-3 } \\
\text { mse-miR-6097 } \\
\text { mse-miR-6100 } \\
\text { mse-miR-9570 } \\
\text { mse-miR-9571a } \\
\text { mse-miR-9571b } \\
\text { t357780 } \\
\text { t454580 }\end{array}$ & $\begin{array}{c}27 \\
27 \\
27 \\
2 \\
13 \\
2 \\
34 \\
46 \\
4\end{array}$ & $\begin{array}{c}87 \\
87 \\
87 \\
32 \\
35 \\
2 \\
35 \\
45 \\
\\
2\end{array}$ & $\begin{array}{c}40 \\
40 \\
40 \\
1 \\
\\
3 \\
46 \\
72 \\
1 \\
1\end{array}$ & $\begin{array}{c}1 \\
1 \\
1 \\
47 \\
1\end{array}$ & $\begin{array}{c}2 \\
2 \\
2 \\
125 \\
2 \\
1\end{array}$ & $\begin{array}{c}1 \\
1 \\
1 \\
23\end{array}$ \\
\hline $\begin{array}{l}\text { t2290056 } \\
\text { t3057689 } \\
\text { t3301296 } \\
\text { t4742895 } \\
\text { mse-bantam } \\
\text { mse-miR-iab-4 } \\
\text { mse-let-7a }\end{array}$ & $\begin{array}{c}1 \\
13 \\
23512 \\
1362 \\
288 \\
177249 \\
\end{array}$ & $\begin{array}{c}2 \\
1 \\
110 \\
12103 \\
2291 \\
2 \\
94886\end{array}$ & $\begin{array}{c}1 \\
26 \\
7404 \\
1009 \\
90 \\
79507 \\
\end{array}$ & $\begin{array}{l}28 \\
29\end{array}$ & $\begin{array}{l}21 \\
89\end{array}$ & $\begin{array}{c}8 \\
10\end{array}$ \\
\hline
\end{tabular}

* Abundances are shown as normalized reads per million (NRPM). Numbers are blank for those either non-detectable or with normalized values below 0.5 .

Table 4. Predicted hemocyte-specific targets

\begin{tabular}{ll}
\hline \multicolumn{1}{c}{ gene } & \multicolumn{1}{c}{ miRNA } \\
\hline aminoacylase & mse-miR-11* \\
IML-3a & $\mathrm{t} 3301296$ \\
lacunin & $\mathrm{t} 3301296$ \\
lectin & mse-miR-11, 6096-1, 6096-2, 6096-3 \\
proPO-1 & mse-miR-11, 190, bantam* \\
serpin 2 & mse-miR-6093* \\
\hline
\end{tabular}

Table 5. Predicted fat body-specific targets *

\begin{tabular}{|c|c|}
\hline miRNA & putative targets \\
\hline mse-miR-1 & $\begin{array}{l}\text { 2-deoxyglucose-6-phosphate phosphatase, 6-phosphogluconate dehydrogenase, ADCY type 9, AMP deaminase } \\
\text { 2, elF4A, Innexin, malate dehydrogenase, } \mathrm{Na}(+) / \mathrm{H}(+) \text { hydrogen antiporter } 1 \text {, PABP-interacting protein 1, phospho- } \\
\text { ribosyl pyrophosphate synthetase, phosphoribosylformylglycinamidine synthase, } 5 \text {-aminoimidazole-4-carboxamide } \\
\text { ribonucleotide formyltransferase, Kelch-like protein } 10 \text {, long chain fatty acid elongase, sugar transporter (m.65628), } \\
\text { sugar transporter (m.197206), trehalose-6-phosphate synthase }\end{array}$ \\
\hline mse-miR-8 & $\begin{array}{l}\text { alanine-glyoxylate aminotransferase, Asn synthetase, endothelin converting enzyme, karmoisin, } \mathrm{Na}(+) / \mathrm{H}(+) \text { hydro- } \\
\text { gen antiporter } 1 \text {, phosphoribosylformylglycinamidine synthase, prenyl-dependent CAAX metalloprotease, cuticle } \\
\text { protein (m.22182), mitochondrial carrier protein, procollagen-lysine-2-oxoglutarate 5-dioxygenase, salivary purine } \\
\text { nucleosidase, seminal fluid protein CSSFP048, serine protease-like protein } 4 \text {, sugar transporter } 4\end{array}$ \\
\hline mse-miR-10a & $\begin{array}{l}\text { ATP citrate lyase, Cameo2, cytochrome B5, elF4A, fusilli isoform G, glycogen synthase, NIPSNAP protein, PAP1, } \\
\text { Phe hydroxylase, hypoxia inducible factor } 1 \text {, Ras-related protein rab-39b-like, SLY-1 homolog, syndecan, UDP- } \\
\text { glycosyltransferase (m.51342) }\end{array}$ \\
\hline mse-miR-12 & $\begin{array}{l}\text { ADCY type 9, Cameo2, carbonic anhydrase, DNA supercoiling factor, elongase, FAD-dependent oxidoreductase, } \\
\text { glycine cleavage system P, glycine N-methyltransferase like, glycogen synthase, HP6, plexin A, protein disulfide } \\
\text { isomerase, 2-oxoacid dehydrogenases acyltransferase, ecdysone oxidase, serpin 3, sugar transporter (m.154929), } \\
\text { Tolloid-like protein 2, translocation protein SEC62 }\end{array}$ \\
\hline mse-miR-33 & $\begin{array}{l}\text { alanine-glyoxylate aminotransferase, AMP deaminase } 2 \text {, branched-chain } \mathrm{AA} \text { aminotransferase, cytochrome } \mathrm{P} 450 \\
\text { (m.17726), hemolymph JH binding protein, HP21, immunolectin } \mathrm{A}, \mathrm{Na}(+) / \mathrm{H}(+) \text { hydrogen antiporter } 1 \text {, ovary } \\
\text { C/EBPg TF, prenyl-dependent CAAX metalloprotease, Ras-related protein rab-39b-like, SLY-1 homolog }\end{array}$ \\
\hline mse-miR-79 & $\begin{array}{l}\text { adenosylhomocysteinase, brain chitinase and chia, cytochrome } \mathrm{P} 450(\mathrm{~m} .236105) \text {, disulfide isomerase, elF } 4 \mathrm{~A} \text {, } \\
\text { elongase, IML-3, innexin, karmoisin, } \mathrm{Na}(+) / \mathrm{H}(+) \text { hydrogen antiporter } 1 \text {, purine nucleoside phosphorylase, serpin } 4\end{array}$ \\
\hline mse-miR-92a & $\begin{array}{l}\text { ADCY type } 9, \alpha \text {-mannosidase II, colmedin, dorsoventral patterning protein tolloid, glutathione S-transferase } \Omega 3 \text {, } \\
\text { guanine deaminase, JH esterase, molybdopterin synthase catalytic subunit } 2 \text {, nicotinamide riboside kinase } 1 \text {, Phe } \\
\text { hydroxylase, } 5 \text {-aminoimidazole-4-carboxamide ribonucleotide formyltransferase, long chain fatty acid elongase, } \\
\text { secreted peptide } 30 \text {, seminal fluid protein CSSFP048, sugar transporter (m.154929) }\end{array}$ \\
\hline mse-miR-92b & $\begin{array}{l}\alpha \text {-mannosidase II, D-3-phosphoglycerate dehydrogenase, fatty acid synthase, glutathione S-transferase } \Omega 3 \text {, } \\
\text { glycine cleavage system h, glycine N-methyltransferase like, HSP60, IML-3, JH esterase, lipid storage droplet } \\
\text { protein, molybdopterin synthase catalytic subunit 2, myostatin, nicotinamide riboside kinase 1, ovary C/EBPg TF, } \\
\text { S-adenosylmethionine synthase, seminal fluid protein CSSFP048, SLY-1 homolog, sugar transporter (m.154929) }\end{array}$ \\
\hline
\end{tabular}


elF4A, endothelin converting enzyme, gloverin, glycogen synthase, karmoisin, $\mathrm{Na}(+) / \mathrm{H}(+)$ hydrogen antiporter 1 , nicotinic acetylcholine receptor subunit $\alpha$, purine nucleoside phosphorylase, serpin 13 , sugar transporter (m.197206), Tolloid-like protein 2

acyl-CoA $\Delta 9$ desaturase (m.46170), adipokinetic hormone receptor, $\beta$-hexosaminidase (m.22649), Cameo2, cGMP-dependent protein kinase, collagen $\alpha 1$ (IV) chain, endothelin converting enzyme, glutathione S-transferase mse-miR-263a $\Omega 3$, innexin, malate dehydrogenase, nicotinic acetylcholine receptor subunit $\alpha$, purine nucleoside phosphorylase, seminal fluid protein CSSFP011 isoform 1, seminal fluid protein CSSFP048, serpin 3, serpin 4, syndecan, TNFSF13, Xanthine dehydrogenase

mse-miR-263b acetyl-CoA carboxylase, $\beta$-hexosaminidase (m.22649), DNA supercoiling factor, DsCAM, elF4A, IML-3, metalloprotease, UDP-glucose pyrophosphatase AMP deaminase 2, Asn-tRNA synthetase, ATP citrate lyase, chondroitin 4-sulfotransferase, Cys synthase, elF4A, endothelin converting enzyme, glycine N-methyltransferase like, HSP60, JH esterase, karmoisin, multiple coagulation factor deficiency protein 2 like, NIPSNAP protein, PABP-interacting protein 1, phosphoserine phosphatase, Pro-4-hydroxylase $\alpha$ subunit, aldehyde dehydrogenase (m.175855), f-spondin, hypoxia inducible factor 1 , long chain fatty acid elongase, procollagen-lysine-2-oxoglutarate 5-dioxygenase, tyrosine protein kinase, Ras-related protein rab-39b-like, S-adenosyl-Met synthase, serpin 4, transaldolase, UDP-glucose pyrophosphatase

mse-miR-278 mse-miR-279b mse-miR-279c mse-miR-279d mse-miR-281 mse-miR-282 mse-miR-306

mse-miR-308

mse-miR-929b mse-miR-989

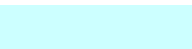

mse-miR-2763

(1)
oxidase (m.79792), hemicentin-1, homo-Cys S-methyltransferase (m.78459), serpin, Ras-related protein rab-39blike, SLY-1 homolog, small HSP, syndecan, Tolloid-like protein 2, transaldolase, trypsin like proteinase, UDPglucose pyrophosphatase, Xanthine dehydrogenase acyl-CoA $\Delta 9$ desaturase (m.106622), adenosylhomocysteinase, apolipoprotein of lipid transfer particle III, branched-chain AA aminotransferase, dihydropteridine reductase, elongase, fusilli isoform $\mathrm{G}$, hemolymph $\mathrm{JH}$ mse-miR-2768 binding protein, hemolymph protein, PABP-interacting protein 1, PAP1, Phe hydroxylase, plexin A, aldehyde dehydrogenase (m.175855), CRAL/TRIO domain-containing protein, cuticle protein (m.169920), sugar transporter (m.197206), UDP-glucose pyrophosphatase

t4742895 6-phosphogluconolactonase, ABC transporter, disulfide isomerase, elF4A, Fkbp13, myostatin, cuticle protein (m.22182), SLY-1 homolog, Taurine catabolism dioxygenase

mse-let-7a kynurenine formamidase, serpin 4 , small HSP acetyl-CoA carboxylase, adipokinetic hormone receptor, trehalose transporter Tret1, karmoisin, lebocin-like protein mse-miR-iab-4 $\mathrm{B}$, nicotinic acetylcholine receptor subunit $\alpha$, phosphatidylethanolamine binding protein, prenyl-dependent CAAX metalloprotease, $\alpha-\mathrm{N}$-acetyl glucosaminidase, homo-Cys S-methyltransferase (m.156992), scavenger receptor class $\mathrm{B}$, small GTP binding protein RAB8 adipokinetic hormone receptor, $\alpha$-mannosidase II, disulfide isomerase, elongase, galactokinase like, $\gamma$-glutamyl mse-miR-8* cyclotransferase like venom protein, glutathione S-transferase $\Omega 3$, glycogen synthase, $\mathrm{Na}(+) / \mathrm{H}(+)$ hydrogen antiporter 1, PABP-interacting protein 1, trehalose-6-phosphate synthase

AMP deaminase 2, branched-chain AA aminotransferase, Cameo2, DNA supercoiling factor, elF4A, hemicentinlike protein 2, hemolymph protein, lactoylglutathione lyase, L-xylulose reductase, purine nucleoside phosphorylase, homo-Cys S-methyltransferase (m.156992), procollagen-lysine-2-oxoglutarate 5-dioxygenase, small HSP, Tolloidlike protein 2 
mse-miR-9b*

mse-miR-10a

mse-miR-92b*

mse-miR-278*

mse-miR-281*

t4742895*
Cameo2, DNA supercoiling factor, elF4A, Hemicentin-like protein 2, Hemolymph protein, HP21, Lactoylglutathione lyase, L-xylulose reductase, NIPSNAP protein, purine nucleoside phosphorylase, homo-Cys S-methyltransferase (m.156992), procollagen-lysine-2-oxoglutarate 5-dioxygenase, Tolloid-like protein 2

ADCY type 9, aldehyde oxidase 2, metalloprotease, molybdopterin synthase catalytic subunit 2, phosphoglucomutase, f-spondin, tricarboxylate transport protein, TNFSF13, translocation protein SEC62

myostatin, $\mathrm{Na}(+) / \mathrm{H}(+)$ hydrogen antiporter 1 , neurotrimin, plexin $\mathrm{A}$, purine nucleoside phosphorylase, taurine catabolism dioxygenase

fusilli isoform $\mathrm{G}, \gamma$-glutamyltransferase

PAP1, cuticle protein (m.22182), hemicentin-1, serpin 4, small GTP binding protein RAB8

Asn synthetase, DsCAM, fusilli isoform $\mathrm{G}$, hemolymph protein, L-xylulose reductase, Kelch-like protein 10 , mitochondrial carrier protein

*If gene names are the same, the transcript IDs (m. number) were included in the parentheses.

Table 6. Predicted midgut-specific targets *

miRNA

mse-miR-1

mse-miR-10a

mse-miR-137

mse-miR-263a

mse-miR-263b

mse-miR-277

mse-miR-281

\section{me}

mse-miR-283

mse-miR-316

mse-miR-750

mse-miR-6094

mse-miR-6094

mse-miR-9571a

putative targets

adenylsulfate kinase, calmodulin, copper transporter, HoxX, hydroxybutyrate dehydrogenase (m.24658), isochorismatase domain containing protein, lipase, mitochondrial succinate semialdehyde dehydro-genase, protein mesh, $\alpha$-1,3-fucosyltransferase, glucocerebrosidase isoform 1, mannose-1-phosphate guanylyltransferase, selenoprotein M, vacuolar ATP synthase subunit S1, pyridoxal-phosphate dependent enzyme, pyridoxine 5'-phosphate oxidase, regucalcin, renin receptor like isoform 1, RING finger protein 181, serine protease (m.165170), sideroflexin, sterol carrier protein 2/3-oxoacyl-CoA thiolase, sugar transporter (m.45569), synaptobrevin, tetraspanin1, vacuolar ATP synthase $21 \mathrm{kDa}$ proteolipid subunit, v-type proton ATPase subunit C, v-type proton ATPase subunit D, v-type proton ATPase subunit E, v-type proton ATPase subunit $F$, v-type proton ATPase subunit $G$

adenylate cyclase, elF2A, GP40, isovaleryl CoA dehydrogenase, metal transporter CNNM2 like, peripheral-type benzodiazepine receptor, protein canopy like, synaptic vesicle protein, SSR $\beta$ subunit

4-nitrophenylphosphatase, ACBP, Acyltransferase, ADGF-like, Bombyrin precursor, CCDC, cytosolic malate dehydrogenase, enoyl CoA hydratase, glucose dehydrogenase, glutathione S-transferase 13, isocitrate dehydrogenase (m.30761), pancreatic lipase, prenyl-dependent CAAX metalloprotease, bhlhzip TF bigmax, carboxypeptidase A like, dipeptidyl peptidase, mitochondrial short-chain specific acyl-CoA dehydrogenase, zinc finger protein, pyroglutamyl peptidase 1, sugar transporter (m.222220), sugar transporter SWEET1, TPPP family protein, trypsin-like protease, v-type proton ATPase subunit D

2-deoxyglucose-6-phosphate phosphatase, acyl-CoA dehydrogenase, cadherin, carboxypeptidase B, dimeric dihydrodiol dehydrogenase, enoyl CoA isomerase, MCT (m.228787), nitrilase, protein mesh, RING finger and WD repeat domain-containing protein 3 , sugar transporter (m.45569)

AMP dependent CoA ligase, ecdysoneless, glucose dehydrogenase, HSP90 $\beta$, metal transporter CNNM2 like, protein cueball, amidase, bhlhzip TF bigmax, cAMP-dependent protein kinase catalytic subunit, lysosomal acid lipase, pyroglutamyl peptidase 1 , retinol dehydrogenase

2-hydroxyphytanoyl-CoA lyase, ACBP homolog, AMP dependent CoA ligase, bombyrin precursor, chorion b-ZIP TF, cytochrome P450 (m.45481), flavin reductase, HSP105, inorganic pyrophosphatase, isocitrate dehydrogenase (m.30761), maggie, mitochondrial succinate semialdehyde dehydrogenase, peripheral-type benzodiazepine receptor, phosphotriesterase like, prohibitin WPH, protein cueball, $\alpha-1,3-$ fucosyltransferase, carbonic anhydrase, pyroglutamyl peptidase 1, regucalcin, SCOT, Sel1 repeat-containing protein 1 like, serpin $15 \mathrm{~A}$, sphingolipid $\Delta 4$-desaturase, TPPP family protein, transporter (m.87506), v-type proton ATPase subunit B 3-dehydroecdysone-3alpha reductase, carboxypeptidase 3, CRAL-TRIO domain-containing protein, cytochrome P450 6AE32, elF2A, glutamate receptor, inorganic pyrophosphatase, isochorismatase domain containing protein, LRR, NADPH cytochrome P450 reductase, serpin 15A

acyltransferase, adenylsulfate kinase, $\alpha$-amylase, aminoacylase 1 , aminopeptidase $\mathrm{N}-11$, Bombyrin precursor, calmodulin isoform A, copper transporter, cytosolic malate dehydrogenase, ER lumen protein retaining receptor, FAMeT, fatty acid binding protein, glutamate receptor, GP40, hydroxysteroid dehydro-genase, isocitrate dehydrogenase (m.61421), kynureninase, LPP1, MSBP, peritrophic membrane chitin binding protein, alcohol dehydrogenase, cAMP-dependent protein kinase catalytic subunit, carbonic anhydrase, pyridoxine 5'-phosphate oxidase, SCP related protein, short chain type dehydrogenase (m.29729), sphingolipid $\Delta 4$-desaturase, sugar transporter (m.222220), synaptobrevin, trypsin alkaline D, trypsin-like protease, v-type proton ATPase subunit D 3-dehydroecdysone-3 $\alpha$ reductase, Arg kinase, calmodulin isoform A, cytochrome b5, GP40, hatching enzyme like protein, hydroxybutyrate dehydrogenase (m.198924), kynureninase, LAP, mitochondrial cytochrome C oxidase subunit 6A, phosphoenolpyruvate synthase, alcohol dehydrogenase, regucalcin, SSR, succinyl-CoA ligase $\beta$ chain, Toll9, TP53-regulated inhibitor of apoptosis 1-B organic cation transporter (m.212782), Pap-inositol-1,4-phosphatase, sodium-bile acid co-transporter, V-type proton ATPase subunit B

carboxyl cholinesterase, pancreatic lipase, peripheral-type benzodiazepine receptor, Ras family protein, short chain type dehydrogenase (m.29729), vacuolar ATPase subunit M9.7

2-deoxyglucose-6-phosphate phosphatase, ACBP, adrenodoxin, Bombyrin precursor, calcium-binding protein p22, copper transporter, cytosolic JH binding protein 36 kDa subunit, GP40, HoxX22, lipid storage droplet protein 2, mitochondrial carrier like, mitochondrial NADPH adrenodoxin oxidoreductase, neuro-peptide receptor A10, Pap-inositol-1,4-phosphatase, peptidyl-prolyl cis-trans isomerase, peroxisomal biogenesis factor 19, phosphoenolpyruvate synthase, bhlhzip TF bigmax, mitochondrial short-chain specific acyl-CoA dehydrogenase, Ras family protein, RGS-GAIP interacting protein GIPC, SCP related protein, sugar transporter SWEET1, transferrin3, trypsin-like protease, v-type proton ATPase subunit $F$ 
2-deoxyglucose-6-phosphate phosphatase, ACBP, adrenodoxin, Bombyrin precursor, B. mori glutathione-Stransferase 4, B. mori glutathione-S-transferase 5, calcium-binding protein p22, carbonyl reductase, chitin binding domain 3 protein, copper transporter, fatty acid binding protein, glutathione S-transferase 13, His-triad nucleotide binding protein 3 , hydroxybutyrate dehydrogenase $(\mathrm{m} .24658)$, isocitrate dehydrogenase $(\mathrm{m} .30761)$, mse-miR-9571b kynureninase, MCT (m.94739), mitochondrial carrier like, MSBP, Pap-inositol-1,4-phosphatase, peripheral-type benzodiazepine receptor, $\alpha$-1,3-fucosyltransferase, bhlhzip TF bigmax, lysosomal acid lipase, RAB6-interacting golgin like, vacuolar ATP synthase subunit S1, pyroglutamyl peptidase 1, Ras family protein, SCP related protein, serine protease (m.46246), short chain type dehydrogenase (m.81137), sugar transporter SWEET1, synaptic vesicle glycoprotein $2 \mathrm{~B}$, synaptobrevin, transporter (m.78218), v-type proton ATPase subunit $\mathrm{F}$ chitin binding domain 3 protein, citrate synthase, cytochrome P450 4M1, ecdysoneless, glucose dehydrogenase, mse-miR-9a* HoxX, lactoylglutathione lyase, LRR, metal transporter CNNM2 like, methylmalonyl-CoA carboxyltransferase 12S subunit, organic cation transporter (m.57266), peroxisomal biogenesis factor 19, harmonin, SCP related protein

mse-miR-10a*

mse-miR-281*

mse-miR-283*

mse-miR-316*

mse-miR-750* glutathione S-transferase 13, HoxX22, methylcrotonoyl-CoA carboxylase $1 \alpha$, NADPH cytochrome P450 reductase, dipeptidyl peptidase, SSR $\beta$ subunit

cytochrome c-like, isochorismatase domain containing protein, phosphoenolpyruvate synthase, bhlhzip TF bigmax

3-dehydroecdysone-3 $\alpha$ reductase, elF2A, O-acyltransferase, peroxisomal biogenesis factor 19, prenyl-dependent CAAX metalloprotease, bhlhzip TF bigmax, pyridoxal-phosphate dependent enzyme

acetoacetyl-CoA thiolase, ARF, B. mori glutathione-S-transferase 4, cytochrome P450 6AE32, fatty acid binding protein, glutathione S-transferase 13 , HoxX, innexin, isovaleryl CoA dehydrogenase, metal transporter CNNM2 like, ADK 3, regucalcin, retinol dehydrogenase, short chain type dehydrogenase (m.29729), v-type proton ATPase subunit C

dimeric dihydrodiol dehydrogenase, lactoylglutathione lyase, methylcrotonoyl-CoA carboxylase $1 \alpha$, NADPH cytochrome $\mathrm{P} 450$ reductase

*If gene names are the same, the transcript IDs (m. number) were included in the parentheses.

Table 7. Potential regulatory pairs of miRNAs and mitochondrial genes

\begin{tabular}{ll}
\hline \multicolumn{1}{c}{ Gene } & \multicolumn{1}{c}{ miRNA } \\
\hline mitochondrial ornithine transporter & mse-miR-100 \\
mitochondrial carrier protein & mse-miR-8, t4742895* \\
mitochondrial solute carrier & mse-miR-279c \\
tricarboxylate transport protein & mse-miR-10a* \\
adrenodoxin & mse-miR-9571a, 9571b \\
mitochondrial cytochrome C oxidase subunit 6A & mse-miR-316 \\
mitochondrial NADPH adrenodoxin oxidoreductase & mse-miR-9571a \\
mitochondrial succinate semialdehyde dehydrogenase & mse-miR-1, 277 \\
mitochondrial short-chain specific acyl CoA dehydrogenase & mse-miR-137, 9571a \\
SCOT & mse-miR-277 \\
mitochondrial carrier like & mse-miR-9571a, 9571b \\
\hline
\end{tabular}

\section{Figure legends}

Fig. 1. Length distributions of frequencies for total (red bars, left y-axis) and unique (black bars, right $y$-axis) reads in the three libraries combined.

Fig. 2. Predicted fold-back structures of novel M. sexta miRNA precursors. Mature sequences are shown in red bold capital letters.

Fig. 3. Eight clusters of $M$. sexta miRNA profiles with precursors identified. The color scale of $\log _{2} \mathrm{NC}$ is shown on the bottom right. $\log _{2} 0.5($ i.e. -1$)$ is represented by black. F, $\mathrm{H}$ and $\mathrm{G}$ stands for fat body, hemocytes and midgut, respectively. 
Fig. 1

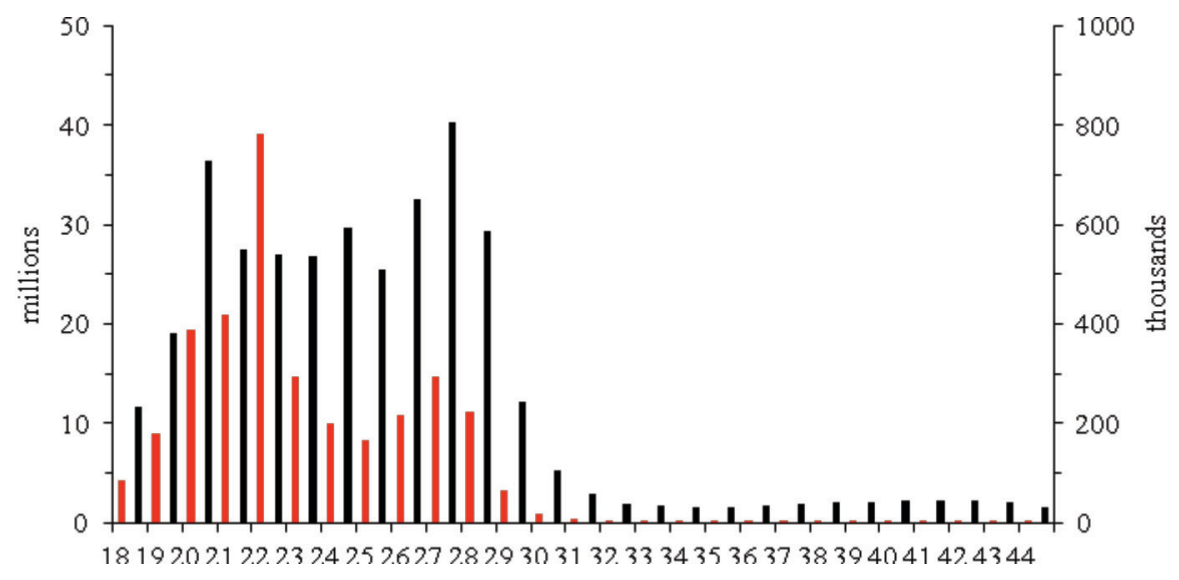

Fig. 2
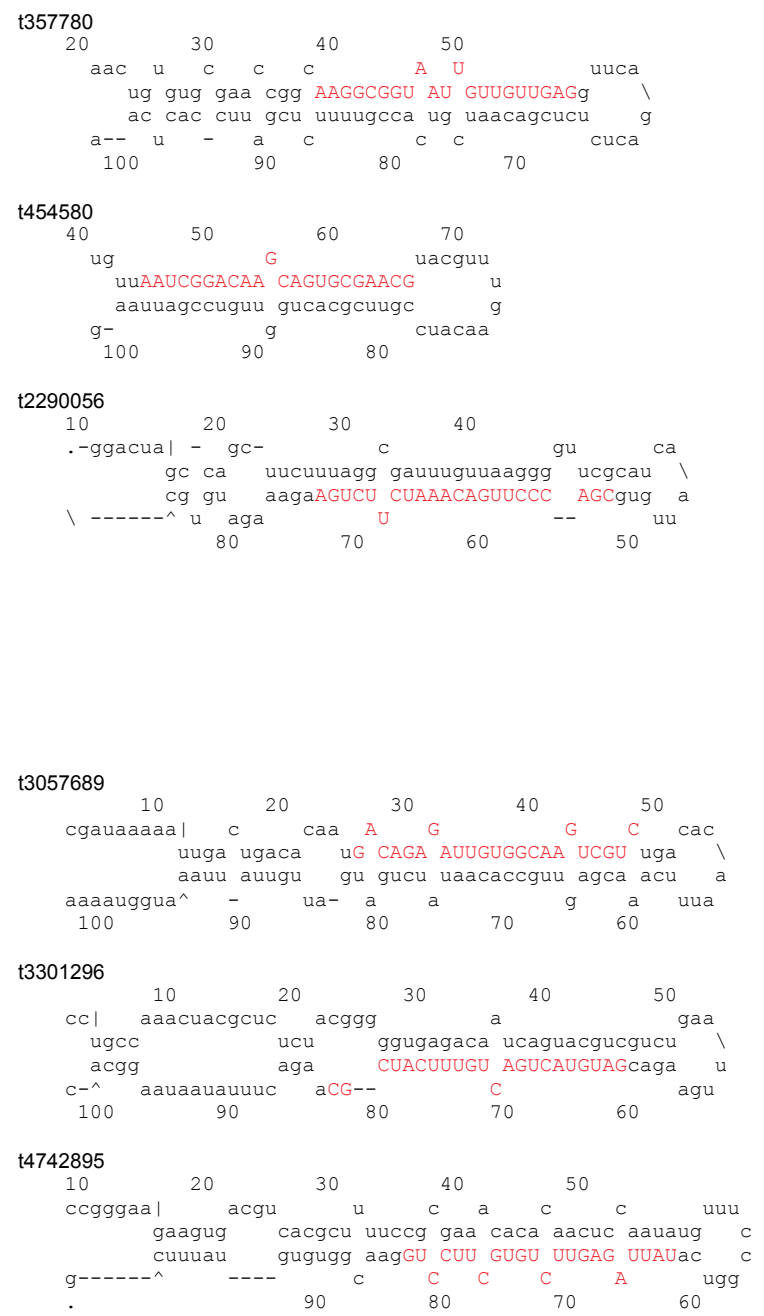
Fig. 3

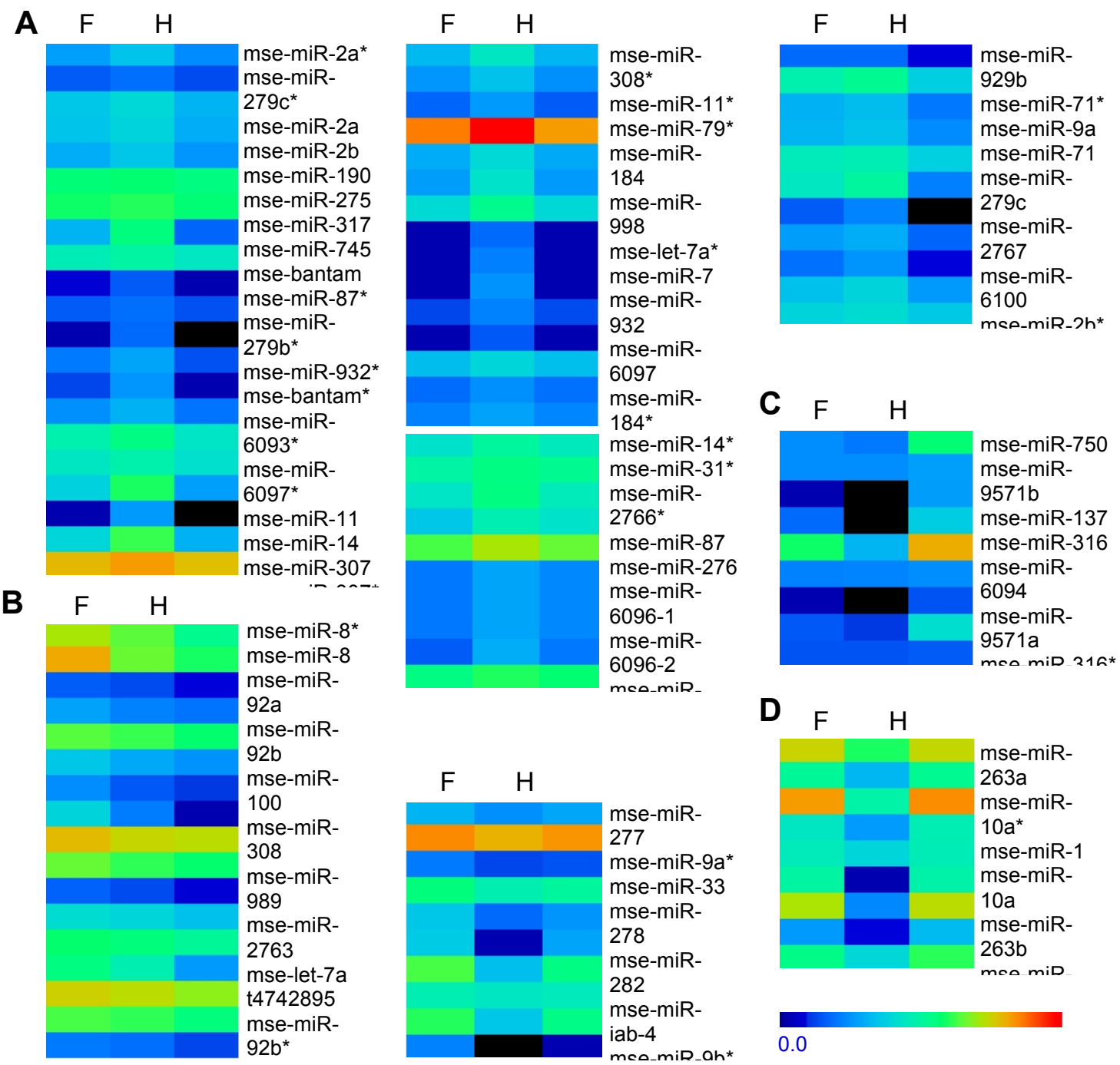


Graphical Abstract

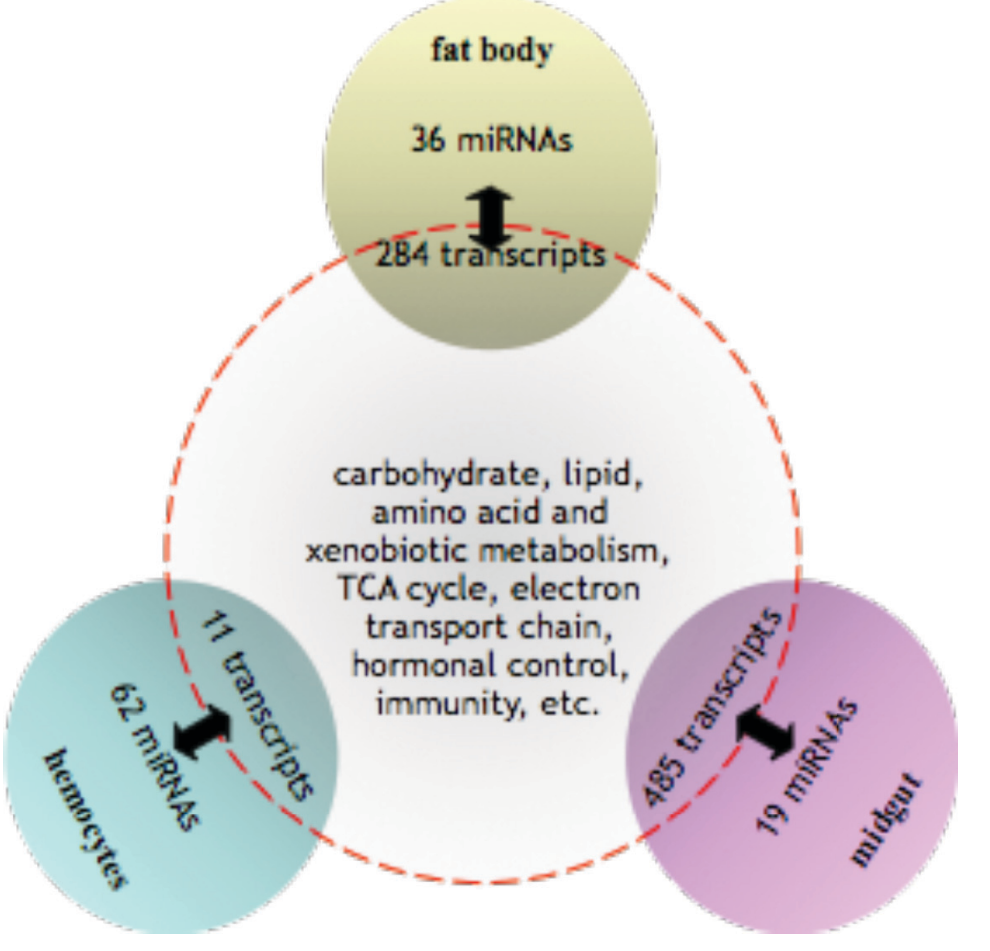

